\title{
Adjuvant therapy for unresectable meningiomas: benign and malignant
}

\author{
Thomas C. Chen, M.D., Ph.D., ${ }^{1}$ And Marc C. Chamberlain, M.D. ${ }^{2}$ \\ ${ }^{I}$ Surgical Neurooncology, University of Southern California, Los Angeles, California; \\ and ${ }^{2}$ Fred Hutchinson Research Institute, University of Washington, Seattle, Washington
}

$\mathrm{M}$ ENINGIOMAS are the most common form of extraaxial primary brain tumor and are discovered not infrequently in otherwise asymptomatic patients. The authors of natural history observational studies have suggested that the majority of asymptomatic meningiomas grow at a slow incremental rate and therefore may be reasonably followed without intervention. Nonetheless, a substantial number of them become symptomatic and are treated surgically. Not all meningiomas, however, can be safely or completely resected, and adjuvant therapeutic intervention is often warranted. This issue of Neurosurgical Focus is directed at aggressive or malignant meningiomas, as well as ones that cannot be completely excised. Our goal is to present current knowledge of meningiomas and highlight potential new therapies.

The symposium opens with a general overview of intracranial meningiomas by Chamberlain and is followed by a discussion of epidemiology and etiopathogenesis of meningiomas by Barnholtz-Sloan. Commins reviews the pathological nature and classification of meningiomas. Rogers next discusses the use of conventional externalbeam radiotherapy in the management of these lesions. Loeffler and colleagues summarize their extensive experience with stereotactic radiotherapy for benign meningiomas. Davidson then presents a single-institution perspec- tive on the utility of Gamma Knife surgery for meningiomas of the cranial base.

In the next section of the meningioma symposium, the authors discuss medical management of meningiomas beginning with Couldwell's paper regarding the use of cyclooxygenase inhibition with COX-2 agents. Roser discusses the eicosanoid cascade as a target for meningioma chemotherapy, and this is followed by Jensen's discussion of calcium channel antagonists as inhibitors of meningioma proliferation. Chen presents laboratory data demonstrating that the protease inhibitor, nelfinavir, has at least additive cytotoxicity when combined with imatinib. Newton next summarizes the literature regarding hydroxyurea as a chemotherapeutic agent for meningiomas, and this is followed by Wen's eloquent review of targeted therapy and potential strategies for the treatment of meningioma.

The symposium concludes with two special reviews: intraosseous meningiomas reviewed by Elder and intraspinal meningiomas summarized by Vrionis.

As editors of this issue of Neurosurgical Focus highlighting the treatment of meningiomas, we would like to thank the contributors for their timely and thoughtful reviews, and hope that this symposium will contribute to an improved understanding and appreciation of new and evolving therapies for meningiomas. 